\title{
NUTRIÇÃO E PRODUÇÃO DA BANANEIRA 'PRATA- ANÃ' ADUBADA COM ZINCO E BORO DIRETAMENTE NO RIZOMA, VIA MUDA DESBASTADA ${ }^{1}$
}

\author{
MARIA GERALDA VILELA RODRIGUES ${ }^{2}$, CARLOS RUGGIERO $^{3}$, \\ WILLIAM NATALE ${ }^{4}$, DILERMANDO DOURADO PACHECO 5
}

RESUMO - Com o objetivo de avaliar o efeito de diferentes doses de Zn e B, aplicados diretamente no rizoma, via muda desbastada, sobre a nutrição das plantas e produção da bananeira 'Prata-anã', foi instalado um experimento em Jaíba - MG, sob irrigação, conduzido por três ciclos produtivos consecutivos. Utilizou-se o desbastador "lurdinha" para extrair a gema apical de um broto cortado rente ao solo, de forma que, no local, ficasse um orifício onde os adubos foram aplicados. Empregou-se o delineamento experimental inteiramente casualizado, com 20 tratamentos resultantes de um fatorial completo entre 5 doses de zinco $\left(0 ; 2,5 ; 5,0 ; 7,5\right.$ e 10,0 g de Zn família ${ }^{-1}$ ano $\left.{ }^{1}\right)$ x 4 doses de boro $\left(0 ; 0,68 ; 1,36\right.$ e 2,04 $\mathrm{g} \mathrm{B}$ família $^{-1}$ ano $\left.^{-1}\right)$, com 10 repetições de uma planta. Avaliaram-se os teores de nutrientes na terceira folha e a produção em massa de frutos por cacho. Os adubos promoveram alterações nos teores foliares de nutrientes, porém sem magnitude suficiente para alterar a condição nutricional quando se consideram as faixas de suficiência. O Zn interferiu na produção, porém os menores valores foram observados na dose intermediária e não houve ajuste de modelo de regressão.

Termos para indexação: adubação, micronutrientes, Musa, teores foliares

\section{'PRATA ANÃ' BANANA PLANT NUTRITION AND PRODUCTION FERTILIZED WITH ZINC AND BORON VIA RHIZOMA}

\begin{abstract}
It was installed one experiment in Jaíba-MG in a 6.6 year-old banana plantation, conducted for three consecutive productive cycles, aiming to evaluate the effect of different proportions of zinc and boron applied directly in the cut off seedling, under the nutrition of the plants and in the production of 'Prata Anã' banana plant. It was used the "lurdinha" cut off to extract the apical vital point of a germination, which was cut near the soil, in such a way that it remained an opening where the fertilizer was applied. It was utilized the entirely casual experimental delineation, with 10 repetitions of one plant, and with 20 resultant treatments of a complete factorial between 5 proportions of Zinc $\left(0 ; 2.5 ; 5.0 ; 7.5\right.$ and $10.0 \mathrm{~g}$ of year ${ }^{-1}$ plant $^{-1}$ Zinc $) \times 4$ proportions of boron $(0 ; 0.68 ; 1.36$ and 2.04 $\mathrm{g}_{\text {year }}{ }^{-1}$ plant $^{-1}$ Boron). The fertilizers promoted some alterations in the foliar contents of nutrients, however it happened without enough magnitude to alter the nutritional condition when it is considered the bands of sufficiency. Although the Zinc interfered in the production, this was in statistics the same when compared to the evidence (zero proportion) and the biggest proportion.
\end{abstract}

Index terms: micronutrients, fertilization, foliar contents, Musa.

\section{INTRODUÇÃO}

A região norte de Minas Gerais possui cerca de 8 mil hectares cultivados com bananeira (Abanorte, 2006), aproximadamente $20 \%$ da área produtora do Estado, basicamente com 'Prata-anã' sob irrigação, principalmente por microaspersão. Teores inadequados de zinco foliar são normalmente observados nas análises de rotina feitas pelo laboratório da Epamig/CTNM (Centro Tecnológico do norte de Minas), mesmo em bananais com altas concentrações de Zn no solo. Quanto ao boro, não raro, são determinados, nas análises foliares, teores inferiores a $10 \mathrm{mg} \mathrm{kg}^{-1}$, tidos como limite mínimo para esse nutriente na cultura.

Segundo López e Espinosa (1995), a nutrição é um fator determinante para que a bananeira produza elevada quantidade de biomassa em um curto período de tempo. Para tanto, são necessárias grandes quantidades de fertilizantes, não só devido à elevada quantidade de nutrientes absorvidos e exportados pelos frutos, como também porque os solos da maioria das regiões produtoras são, geralmente, de baixa fertilidade (Borges et al., 1987).

No Brasil, o boro e zinco são os micronutrientes que se encontram deficientes com maior freqüência em bananeiras (Borges et al., 1987). Em levantamento do estado nutricional dos bananais do norte de Minas Gerais, feito por Silva e Rodrigues (2001), os micronutrientes estavam deficientes na seguinte ordem decrescente: $\mathrm{Zn}>\mathrm{Cu}>\mathrm{Fe}>\mathrm{Mn}>\mathrm{B}$. O Zn apresentou-se deficiente em $72 \%$ das amostras foliares $\left(<20 \mathrm{mg} \mathrm{kg}^{-1}\right)$.

Aplicar o fertilizante no lugar certo é quase tão importante quanto usar a fórmula e a quantidade adequadas (Malavolta,

\footnotetext{
Trabalho (095-06). Recebido em : 20-07-2006. Aceito para publicação em : 04-09-2007. Parte da Tese de Doutorado do primeiro autor, apresentada à FCAV/Unesp, Jaboticabal - SP. Apoio financeiro: FAPEMIG.

${ }^{2}$ Pesquisadora da Epamig/CTNM, CEP 39527-000, Nova Porteirinha - MG, CP. 12. magevr@epamig.br

${ }^{3}$ Professor Titular do Departamento de Produção Vegetal, FCAV/Unesp - Campus de Jaboticabal. CEP 14870-000, Jaboticabal - SP. ruggiero@fcav.unesp.br

${ }^{4}$ Professor Adjunto do Departamento de Solos e Adubos, FCAV/Unesp - Campus de Jaboticabal. natale@fcav.unesp.br

${ }_{5}^{5}$ Pesquisador da Epamig/CTNM, CEP 39527-000, Nova Porteirinha - MG, CP. 12. ddpacheco.agro@gmail.com
} 
1980). Para esse autor, localização certa significa colocar o adubo no solo de maneira tal que, de um lado, o possível dano à planta fique reduzido a um mínimo e, de outro, a cultura tenha maior eficiência de absorção atendendo à sua exigência nutricional.

A bananeira apresenta um caule subterrâneo, do tipo rizoma, no qual estão apoiados todos os seus órgãos: raízes, gemas, rebentos, pseudocaule, folhas e frutos (Soto Ballestero, 2000). No ponto de ligação entre o rizoma da planta-mãe e do rebento, o córtex e o cilindro central apresentam uma região bastante comprimida por onde ocorrem as trocas de seiva e hormônios; portanto, as plantas de uma mesma "família" de bananeiras compõem um único sistema, interligado por seus rizomas (Moreira, 1999). Com base nessa ligação e na troca de material entre as plantas, esse autor propõe a aplicação de fertilizantes no broto desbastado, através de orifícios feitos com o desbastador "lurdinha". Ainda segundo Moreira (1999), o adubo aí colocado entra em contato com o rizoma da planta-mãe e, através dele, é absorvido e passa a circular no sistema de todas as plantas que estiverem interligadas.

Em função do exposto, o objetivo deste trabalho foi avaliar o efeito do fornecimento de diferentes doses de zinco e boro, diretamente no rizoma, via muda desbastada, na nutrição das plantas e na produção da bananeira 'Prata-anã', cultivada sob irrigação por microaspersão.

\section{MATERIAL E MÉTODOS}

O experimento foi conduzido na Empresa de Pesquisa Agropecuária de Minas Gerais (Epamig), no Perímetro Irrigado do Jaíba, município de Jaíba-MG. A região apresenta pluviosidade média de $870 \mathrm{~mm}$ anuais, temperatura média de $24^{\circ} \mathrm{C}$, altitude de $452 \mathrm{~m}$, umidade relativa média de $70 \%$ e clima do tipo Aw, segundo a classificação de Köeppen. O solo da área foi classificado como Latossolo Vermelho-Amarelo distrófico, com 220; 680 e $100 \mathrm{~g} \mathrm{~kg}^{-1}$ de argila, areia e silte, respectivamente, na profundidade de 0-20 $\mathrm{cm}$.

O experimento foi conduzido de fevereiro de 2003 a novembro de 2005, em um bananal de 'Prata anã' de 1,8 ha, implantado em julho de 1996 (portanto, com 6,6 anos), com mudas micropropagadas, no espaçamento 3,0 x 2,7 m, estabilizado e em franca produção. Aárea foi irrigada por microaspersão, com lâmina de água calculada com base na evapotranspiração pelo Tanque Classe "A". As adubações de cobertura foram parceladas mensalmente, calculadas com base na interpretação dos resultados analíticos de amostras de solo e folhas coletadas a cada quatro meses.

Os resultados da caracterização química de amostras do solo $(0-20 \mathrm{~cm})$ coletadas na instalação do experimento, feita segundo Embrapa (1997), foram: 36; 10,3; 37 e $7 \mathrm{mmol} \mathrm{dm}^{-3} \mathrm{de} P$, $\mathrm{K}$, Ca e Mg; além de 0,$3 ; 0,4 ; 20,1 ; 35,9 ; 6,4$ e $0,0 \mathrm{mg} \mathrm{dm}^{-3}$, de B, Cu, $\mathrm{Fe}, \mathrm{Mn}, \mathrm{Zn}$ e Al, respectivamente. $\mathrm{O} \mathrm{pH}\left(\mathrm{H}_{2} \mathrm{O}\right)$ foi 6,$0 ; \mathrm{T}=78$ mmol $\mathrm{dm}^{-3}, \mathrm{e} \mathrm{V}=69 \%$. Utilizou-se extrator Mehlich-1 para $\mathrm{P}, \mathrm{K}$, $\mathrm{Cu}, \mathrm{Fe}, \mathrm{Mn}$ e $\mathrm{Zn}$.

Na instalação do experimento, fez-se também amostragem foliar, conforme recomendado por Silva et al. (1999, 2002), utilizando a terceira folha a contar do topo da planta, coletada de plantas que apresentavam inflorescência com, no máximo, três pencas de flores masculinas abertas, sendo que, dessa folha, se coletou a metade interna (sem a nervura central) de uma faixa de $10 \mathrm{~cm}$ de largura, retirada na parte central da folha. As amostras vegetais foram secas em estufa com circulação forçada de ar, a $65^{\circ} \mathrm{C}$, por 72 horas, e moídas em moinho tipo Willey. Foram determinados os teores de N-total pelo método Kjeldahl (digestão sulfúrica) (Tedesco et al., 1985); P, K, S, Ca, Mg e Zn em extrato nitroperclórico dosados, pelo método da vitamina $\mathrm{C}$ (Braga $\mathrm{e}$ Defelipo, 1974), S por turbidimetria de sulfato, K por fotometria de chama (Blanchar et al., 1965), Ca, Mg e Zn por espectrofotometria de absorção atômica. O B foi obtido por digestão via seca, seguida de dosagem com azometina-H base (Malavolta et al., 1997). Os resultados encontram-se na Tabela 1 .

O experimento constituiu-se da adubação com zinco e boro, aplicados diretamente no rizoma da planta, via muda desbastada, conforme metodologia recomendada por Teixeira (1997) e Moreira (1999 e 2001), utilizando, como fontes, sulfato de zinco (20\% de $\mathrm{Zn})$ e ácido bórico $(17 \%$ de B). Empregou-se o desbastador "lurdinha" para extrair a gema apical de um broto cortado rente ao solo, de forma que, no local, ficasse um orifício de até $7,0 \mathrm{~cm}$ de profundidade (a depender do tamanho da muda) por 2,8 cm de diâmetro, onde os adubos foram adicionados.

Utilizou-se o delineamento experimental inteiramente casualizado, com 10 repetições de uma planta. Os 20 tratamentos foram constituídos por 5 doses de zinco $(0 ; 2,5 ; 5,0 ; 7,5$ e 10,0 g família $^{-1}$ ano $\left.^{-1}\right)$ e 4 doses de boro $\left(0 ; 0,68 ; 1,36\right.$ e 2,04 $\mathrm{g} \mathrm{família}^{-1}$ ano $\left.{ }^{1}\right)$, em fatorial completo. A dose máxima de cada nutriente, equivale a 100\% da recomendação feita para aplicação via solo, conforme Borges et al. (1987) e Silva et al. (1999). As doses anuais referentes aos tratamentos foram divididas em três aplicações, nos meses de fevereiro, junho e outubro, repetindo-as durante os três anos de condução do experimento.

As 200 famílias que constituíram a área útil foram selecionadas por semelhança, apresentando: planta-mãe com cacho, planta-filha na pré-floração e com, no mínimo, dois brotos. Um dos brotos deu seqüência à família (planta-neta), e o outro foi desbastado para receber a aplicação dos tratamentos. Aplicou-se o cálculo de média, desvio-padrão e coeficiente de variação para atestar a semelhança entre as plantas, no início do experimento. A semelhança entre as plantas selecionadas foi confirmada na plantafilha, que apresentou, em média: $3,48 \mathrm{~m}$ de altura ( $\mathrm{s}=0,3 \mathrm{e} \mathrm{CV}=8,5 \%$ ), $105 \mathrm{~cm}$ de perímetro do pseudocaule a $30 \mathrm{~cm}$ do solo $(\mathrm{s}=10,3 \mathrm{e}$ $\mathrm{CV}=9,8 \%$ ) e 15 folhas ( $\mathrm{s}=2,4$ e $\mathrm{CV}=15,9 \%)$.

Utilizaram-se adubações corretivas de 40, 128 e $120 \mathrm{~g}$ planta $^{-1}$ de S, nos anos de 2003, 2004 e 2005, respectivamente, tendo como fontes superfosfato simples e sulfato de magnésio, a fim de elevar o estado nutricional de $\mathrm{S}$ para plantas, tornando o efeito dos tratamentos com $\mathrm{ZnSO}_{4}$ dependente apenas do micronutriente.

Todas as plantas foram avaliadas no estádio de florescimento e de colheita, durante os três ciclos de cultivo, determinando-se a composição mineral das folhas indicadoras, segundo metodologia já descrita, e a massa fresca dos cachos. Os dados de diagnóstico e de produção foram submetidos à análise de variância e, quando o teste $\mathrm{F}$ foi significativo, feita a 
análise de regressão polinomial.

\section{RESULTADOS E DISCUSSÃO}

As concentrações de nutrientes nas folhas, no início do experimento, foram compatíveis com as de plantas nutricionalmente equilibradas, segundo os dados de Malavolta et al. (1997) e Prezotti (1992) (Tabela 1), excetuando-se os dados de S, Fe, B e Zn, abaixo das faixas de suficiência. O baixo teor foliar de Zn é incompatível com a alta concentração no solo, conforme considerado por Alvarez et al. (1999) (Tabela 2), indicando restrição na disponibilidade desse nutriente para as plantas. A condição de baixos teores foliares de $\mathrm{Zn}$, tão comuns na região (Silva e Rodrigues, 2001), associados às altas concentrações de Zn no solo motivaram a instalação do experimento nessa área.

Trabalhando com a cultivar Prata-anã por microaspersão, no norte de Minas Gerais, Silva et al. (2002) estabeleceram as faixas de suficiência dos nutrientes na terceira folha (Tabela 1) e no solo (Tabela 2) de bananais de alta produtividade $\left(>25 \mathrm{tha}^{-1}\right)$. Nas mesmas condições, Pinho (2004) determinou o teor foliar (Tabela 1) e a concentração de Zn no solo (Tabela 2) associados ao melhor equilíbrio nutricional, os quais diferiram daqueles citados por Malavolta et al. (1997) e Prezotti (1992) para o teor foliar (Tabela 1), assim como de Alvarez et al. (1999) para a concentração no solo (Tabela 2). Com base nos resultados apresentados por Silva et al. (2002) e Pinho (2004), a caracterização inicial do experimento indicava alto teor de P; suficiência de $\mathrm{S}$ e $\mathrm{Zn}$; deficiência de B e Fe nas folhas; e baixa concentração de $\mathrm{Zn}$ no solo.

\section{a)Teores foliares de nutrientes}

Observou-se diluição de todos os nutrientes do primeiro para os ciclos seguintes, com exceção do B, resultante do aumento do porte das plantas e do peso do cacho (dados não apresentados). No primeiro ciclo, apenas os teores foliares de Mg estavam um pouco abaixo da faixa de suficiência estabelecida por Silva et al. (2002); porém, além de essa deficiência ter-se mantido (provavelmente induzida pelo excesso de K), surgiram também deficiências de $\mathrm{Zn}$ no segundo ciclo, e de $\mathrm{S}$ e $\mathrm{Zn}$ no terceiro ciclo. No primeiro ciclo, apenas os teores foliares de B e Zn encontravamse no limite mínimo da faixa de suficiência, porém, no segundo ciclo, estavam em tal condição somente os teores foliares de $\mathrm{P}, \mathrm{S}$ e B; e no terceiro ciclo, apenas N, P e B (Tabelas 1; 2 e 3).

Os teores de nutrientes, encontrados nas análises foliares, variaram apenas em reposta às doses de $\mathrm{Zn}$, para: $\mathrm{P}, \mathrm{K}$ e $\mathrm{Zn}$ no primeiro ciclo (Tabela 3); Ca e Mg no terceiro ciclo (Tabela 5). Foi observada interação entre as doses de $\mathrm{Zn}$ e B para os teores foliares de K e B no segundo ciclo; e de S e B no terceiro ciclo de produção avaliado.

As doses de $\mathrm{Zn}$ exerceram efeito quadrático sobre o teor foliar de P quando esse era mais alto (primeiro ciclo) (Tabela $3 \mathrm{e}$ Figura 1). Não foi observado efeito das doses de Zn sobre o teor foliar de $\mathrm{P}$ quando as plantas apresentavam teores foliares de $\mathrm{P}$ mais baixos (segundo e terceiro ciclos). De acordo com Menguel e Kirkby (1987), o efeito da interação P x Zn ocorre apenas na disponibilidade do Zn nos tecidos; entretanto, Marschner (1995) considera que os maiores efeitos dessa interação ocorrem no solo, com redução na difusão de Zn devido à adição de P. Neste experimento, a interação observada no primeiro ciclo deu-se na planta, uma vez que o $\mathrm{Zn}$ não foi fornecido via solo.

$\mathrm{O}$ teor foliar de $\mathrm{K}$ aumentou de forma linear com o aumento das doses de Zn, no primeiro ciclo (Tabela 3 e Figura 1); já no segundo ciclo, houve efeito do $\mathrm{Zn}$ na ausência de B, além de o B ter reduzido o teor foliar de $\mathrm{K}$ quando na ausência de $\mathrm{Zn}$ (Tabelas 4 e 6). Houve efeito de interação dos tratamentos para teores foliares de $\mathrm{S}$ somente no terceiro ciclo, quando esse nutriente estava abaixo da faixa de suficiência (Tabelas 5 e 7). Nas duas maiores doses de $\mathrm{Zn}$, o menor teor foliar de $\mathrm{S}$ foi determinado quando fornecidos $0,68 \mathrm{~g}$ de $\mathrm{B}$.

Houve efeito quadrático das doses de $\mathrm{Zn}$ para teores foliares de $\mathrm{Ca}$ e $\mathrm{Mg}$ somente no terceiro ciclo (Tabela 6, Figura 2). De acordo com Malavolta (1980), a maior parte do Ca é transportada via xilema, e o movimento ascendente dá-se por reações de troca em vasos condutores (mais que por fluxo de massa), sendo deslocado dos sítios de troca por $\mathrm{Mg}, \mathrm{Mn}$ e $\mathrm{Zn}$. Segundo Marschner (1995), excesso de $\mathrm{Zn}$ pode induzir a deficiência de $\mathrm{Mg}$ nas plantas, pela similaridade do raio iônico de ambos.

Não houve efeito dos tratamentos sobre o teor foliar de B no primeiro ciclo. Já no segundo ciclo, observou-se efeito positivo da aplicação de B, além da interação dos tratamentos no segundo e terceiro ciclos. A interpretação desses resultados, porém, foi comprometida pelo alto coeficiente de variação (Tabelas $3 ; 4$ e 5 ). Esse alto coeficiente de variação pode ser parcialmente explicado pelo momento de amostragem em relação à adubação, uma vez que a coleta de folhas é feita de acordo com o momento de floração de cada planta, e a aplicação do fertilizante obedece a um calendário prévio (três parcelas anuais). Portanto, a amostragem de algumas plantas foi realizada em datas mais próximas à adubação que de outras.

Houve resposta linear positiva dos teores foliares de $\mathrm{Zn}$ às doses de $\mathrm{Zn}$, no primeiro ciclo (Tabela 3 , Figura 1), apesar de a folha amostrada não ser a mais adequada para a determinação do nutriente. Segundo Malburg (1988), trabalhando com diferentes amostragens para diagnose nutricional da bananeira 'Prata-anã', a folha vela (coletada de plantas jovens, com 1,5 $\pm 0,25 \mathrm{~m}$ de altura e 4 a 6 folhas totalmente desenroladas) foi a que apresentou maiores teores foliares de Zn. Marchal e Martin-Prével (1971) afirmam que, como o $\mathrm{Zn}$ é ativador de enzimas responsáveis pela síntese do triptofano, os órgãos em pleno crescimento, coletados em plantas no estádio vegetativo, são mais convenientes para a diagnose nutricional desse elemento.

A resposta não-significativa às doses de $\mathrm{Zn}$ no segundo e terceiro ciclos pode, portanto, ser devida à amostragem da terceira folha, recomendada para a diagnose da bananeira (Silva et al., 2002). Apesar dessa limitação, foi utilizando esse tipo de amostragem, além da mesma variedade, cultivada nessa região, que Silva et al. (2002) e Pinho (2004) obtiveram os resultados aqui utilizados como referência.

Segundo Saes e Quaggio (dados não-publicados, citados por Quaggio e Pizza Junior, 2001), 60 dias após a aplicação de $8 \mathrm{~g}$ de $\mathrm{Zn}$ (como sulfato de zinco) na região meristemática do caule 
(rizoma) da bananeira 'Prata-anã', através do orifício deixado pelo desbastador "lurdinha", os teores foliares na planta adulta passaram de 9,2 para 143,7 $\mathrm{mg} \mathrm{kg}^{-1}$. Da mesma forma, nesse mesmo trabalho, 60 dias após a aplicação de $2 \mathrm{~g}$ de $\mathrm{B}$ (como ácido bórico), os teores foliares da planta adulta passaram de 8,6 para $49,8 \mathrm{mg}$ $\mathrm{kg}^{-1}$. Segundo $\mathrm{Saes}^{5}$ (2006), esses altos teores resultaram em fitotoxidade.

Outro ponto que dificulta a interpretação dos dados é a variabilidade deles, resultante do extenso período de colheita. Como foram selecionadas por semelhança, todas as plantas do primeiro ciclo foram colhidas em um período de 6 meses (outubro de 2003 a abril de 2004), porém as diferenças foram acentuandose com o tempo, o que resultou na colheita do segundo ciclo, em 11 meses (junho de 2004 a maio de 2005), assim como o terceiro ciclo (dezembro de 2004 a novembro de 2005). Segundo MartinPrével (1987), em condições equatoriais, o ciclo produtivo da bananeira independe das estações, havendo produção o ano todo, com sobreposição de ciclos. Nessa condição, segundo o autor, a amostragem de todas as plantas, quando alcançam uma fase específica, resultará no aumento do período global de amostragem, e esse tornar-se-á progressivamente mais longo a cada ciclo de crescimento.

\section{Produção}

A massa de frutos do cacho não foi influenciada pelos tratamentos no primeiro ciclo. No segundo e terceiro ciclos, houve efeito das doses de $\mathrm{Zn}$, com valores mínimos na intermediária (Tabelas 8), não havendo ajuste de modelo pela análise de regressão. Esse resultado foi observado apesar de os teores foliares de $\mathrm{Zn}$ do segundo e terceiro ciclos apresentarem-se abaixo da faixa de suficiência definida por Silva et al. (2002). Porém, outras deficiências ocorreram, como de Mg nos três ciclos e de $\mathrm{S}$ no terceiro ciclo, que podem ter limitado a resposta da planta ao $\mathrm{Zn}$.

Malburg (1988), trabalhando com diferentes amostragens para a diagnose nutricional da bananeira 'Prata-anã', observou correlação de produtividade apenas com teor de $\mathrm{Zn}$ determinado na folha vela (coletadas de plantas jovens, com 1,5 $\pm 0,25 \mathrm{~m}$ de altura e 4 a 6 folhas totalmente desenroladas), nas quais o teor foliar diminuiu (de 36,39 para 32,98) à medida que a produtividade também diminuiu (de 16,7 a 21,7 t ha- ano $^{-1}$ para 13 a $15 \mathrm{t} \mathrm{ha}^{-1}$ ano $^{-}$ ${ }^{1}$ ). Nesse mesmo trabalho, a melhor correlação entre teor foliar de B e produtividade foi obtida nas amostras retiradas de plantas no início da floração (amostras coletadas da terceira folha de plantas com inflorescência recentemente lançada, ainda fechada e já inclinada para baixo). Nessa situação o teor foliar diminuiu (de 20,48 para $17,35 \mathrm{mg} \mathrm{kg}^{-1}$ ) à medida que a produtividade também diminuiu (de 16,7 a 21,7 $\mathrm{t} \mathrm{ha}^{-1}$ ano $^{-1}$ para 5 a $8 \mathrm{t} \mathrm{ha}^{-1}$ ano $^{-1}$ ). Essa última amostragem (plantas no início da floração) é a mais semelhante àquela utilizada no presente trabalho, sendo poucos dias mais precoce.
TABELA 1 - Caracterização inicial do bananal utilizado no experimento; faixas de suficiência* e teor ótimo obtido pelo DRIS ${ }^{* *}$, de nutrientes na terceira folha da bananeira, amostrada na floração.

\begin{tabular}{lccccc}
\hline Nutrientes & $\begin{array}{c}\text { Caracterização } \\
\text { inicial do } \\
\text { bananal }\end{array}$ & $\begin{array}{c}\text { Variedade não- } \\
\text { especificada } \\
\text { (Malavolta et al., 1997) }\end{array}$ & $\begin{array}{c}\text { Prata } \\
\text { (Prezotti, 1992) }\end{array}$ & $\begin{array}{c}\text { Prata-anã } \\
\text { (Silva et al., 2002) }\end{array}$ & $\begin{array}{c}\text { Prata-anã } \\
\text { (Pinho, 2004) }\end{array}$ \\
\hline $\mathrm{N}\left(\mathrm{g} \mathrm{kg}^{-1}\right)$ & 29,9 & $27-36$ & $27-36$ & $25-29$ & \\
$\mathrm{P}\left(\mathrm{g} \mathrm{kg}^{-1}\right)$ & 2,4 & $1,8-2,7$ & $1,8-2,7$ & $1,5-1,9$ & \\
$\mathrm{~K}\left(\mathrm{~g} \mathrm{~kg}^{-1}\right)$ & 32,5 & $35-54$ & $30-54$ & $27-35$ & \\
$\mathrm{Ca}\left(\mathrm{g} \mathrm{kg}^{-1}\right)$ & 6,6 & $2,5-12,0$ & $2,5-12,0$ & $4,5-7,5$ & \\
$\mathrm{Mg}\left(\mathrm{g} \mathrm{kg}^{-1}\right)$ & 3,7 & $3,0-6,0$ & $3,0-6,0$ & $2,4-4,0$ & \\
$\mathrm{~S}\left(\mathrm{~g} \mathrm{~kg}^{-1}\right)$ & 1,8 & $2,0-3,0$ & $2,0-3,0$ & $1,7-2,0$ & \\
$\mathrm{~B}\left(\mathrm{mg} \mathrm{kg}^{-1}\right)$ & 8,6 & $10-25$ & $10-25$ & & \\
$\mathrm{Cu}\left(\mathrm{mg} \mathrm{kg}^{-1}\right)$ & 6,8 & $6,0-30,0$ & $6,0-30,0$ & $2,6-8,8$ & \\
$\mathrm{Fe}\left(\mathrm{mg} \mathrm{kg}^{-1}\right)$ & 68,2 & $80-360$ & $80-360$ & $72-157$ & \\
$\mathrm{Mn}\left(\mathrm{mg} \mathrm{kg}^{-1}\right)$ & 561,4 & $200-2000$ & $200-2000$ & $173-630$ & 14,4 \\
$\mathrm{Zn}\left(\mathrm{mg} \mathrm{kg}^{-1}\right)$ & 14,8 & $20-50$ & $20-50$ & $14-25$ & \\
\hline & \multicolumn{5}{c}{}
\end{tabular}

TABELA 2- Classe de interpretação de fertilidade do solo considerada como "bom" (Alvarez et al., 1999) e faixas de suficiência de atributos químicos do solo para bananeira 'Prata-anã' cultivada no norte de Minas Gerais (Silva et al., 2002; Pinho, 2004).

\begin{tabular}{|c|c|c|c|c|c|c|c|}
\hline & $\begin{array}{c}\mathrm{pH} \\
\left(\mathrm{H}_{2} \mathrm{O}\right)\end{array}$ & $\begin{array}{c}\mathrm{P}^{*} \\
\mathrm{mg} \mathrm{dm}^{-3}\end{array}$ & $\mathrm{H}+\mathrm{Al}$ & $\mathrm{Ca}$ & $\begin{array}{r}\mathrm{Mg} \\
\mathrm{Ol}_{\mathrm{c}} \mathrm{dm}^{-3} \text {. }\end{array}$ & $\mathrm{K}^{*}$ & $\begin{array}{c}\text { B } \\
--\mathrm{mg} \mathrm{dm}^{-3}\end{array}$ \\
\hline Alvarez et al. (1999) & $5,5-6,0$ & $20,1-30,0$ & $<50$ & $24,1-40,0$ & $9,1-15,0$ & $1,81-3,07$ & $0,61-0,90 \quad 1,6-2,2^{*}$ \\
\hline $\begin{array}{l}\text { Silva et al. (2002): } \\
\text { - profundidade } 0-20 \mathrm{~cm} \\
\text { - profundidade } 20-40 \mathrm{~cm}\end{array}$ & $\begin{array}{l}5,5-7,5 \\
5,5-7,5\end{array}$ & & $\begin{array}{l}9-27 \\
9-27\end{array}$ & $\begin{array}{l}35-95 \\
35-94\end{array}$ & $\begin{array}{l}6-18 \\
4-16\end{array}$ & $\begin{array}{l}2,30-7,42 \\
2,05-6,39\end{array}$ & \\
\hline $\begin{array}{l}\text { Pinho (2004): } \\
\text { - Mehlich-1. } \\
\text { - DTPA a pH 7,3 }\end{array}$ & & & & & & & $\begin{array}{c}16,7 \\
5,7\end{array}$ \\
\hline
\end{tabular}

* Extrator Mehlich-1. ${ }^{* *}$ Quando 150 a $250 \mathrm{~g} \mathrm{~kg}^{-1}$ de argila

TABELA 3 - Resumo da análise de variância para teores foliares de nutrientes em bananeira 'Prata-anã' (terceira folha amostrada na floração), no primeiro ciclo de avaliação, em reposta à adubação com $\mathrm{B}$ e $\mathrm{Zn}$ ( $\mathrm{g}$ família-1 $^{-1}$ no $^{-1}$ ). Epamig/CTNM, Jaíba - 2006.

\begin{tabular}{|c|c|c|c|c|c|c|c|c|}
\hline \multirow{2}{*}{$\mathrm{Zn}$} & $\mathrm{N}$ & $\mathrm{P}$ & K & $\mathrm{S}$ & $\mathrm{Ca}$ & $\mathrm{Mg}$ & \multirow{2}{*}{\multicolumn{2}{|c|}{$\begin{array}{c}\mathrm{B} \\
----\mathrm{mg} \mathrm{kg}^{-1}-{ }^{2 n} \\
\end{array}$}} \\
\hline & & & -----g & & & & & \\
\hline 0 & 27,08 & 1,70 & 30,32 & 1,79 & 6,87 & 2,34 & 9,94 & 14,33 \\
\hline 2,5 & 26,76 & 1,78 & 31,10 & 1,83 & 6,31 & 2,29 & 10,51 & 14,99 \\
\hline 5,0 & 26,70 & 1,82 & 30,97 & 1,83 & 6,69 & 2,42 & 11,40 & 15,38 \\
\hline 7,5 & 26,64 & 1,81 & 32,11 & 1,83 & 6,52 & 2,32 & 10,46 & 14,95 \\
\hline 10,0 & 26,54 & 1,79 & 31,56 & 1,83 & 6,35 & 2,32 & 10,23 & 15,48 \\
\hline Teste F & $0,950^{\mathrm{NS}}$ & $2,277 *$ & $2,602 *$ & $0,547^{\mathrm{NS}}$ & $1,003^{\mathrm{NS}}$ & $1,358^{\mathrm{NS}}$ & $0,756^{\mathrm{NS}}$ & $2,459^{*}$ \\
\hline DMS (5\%) & 0,822 & 0,100 & 1,623 & 0,106 & 0,919 & 0,157 & 2,446 & 1,127 \\
\hline \multicolumn{9}{|l|}{ B } \\
\hline 0 & 26,76 & 1,81 & 31,31 & 1,84 & 6,39 & 2,36 & 9,96 & 15,09 \\
\hline 0,68 & 26,65 & 1,79 & 31,07 & 1,83 & 6,74 & 2,32 & 10,51 & 15,03 \\
\hline 1,36 & 26,85 & 1,78 & 31,49 & 1,83 & 6,33 & 2,35 & 10,76 & 14,74 \\
\hline 2,04 & 26,71 & 1,75 & 30,99 & 1,79 & 6,73 & 2,30 & 10,79 & 15,26 \\
\hline Teste $\mathrm{F}$ & $0,205^{\mathrm{NS}}$ & $1,388^{\mathrm{NS}}$ & $0,379^{\mathrm{NS}}$ & $0,822^{\mathrm{NS}}$ & $1,092^{\mathrm{NS}}$ & $0,589^{\mathrm{NS}}$ & $0,472^{\mathrm{NS}}$ & $0,708^{\mathrm{NS}}$ \\
\hline DMS (5\%) & 0,692 & 0,084 & 1,366 & 0,089 & 0,773 & 0,132 & 2,059 & 0,949 \\
\hline \multicolumn{9}{|l|}{ B $\times \mathbf{Z n}$} \\
\hline Teste F & $0,835^{\mathrm{NS}}$ & $1,660^{\mathrm{NS}}$ & $1,143^{\mathrm{NS}}$ & $1,029^{\mathrm{NS}}$ & $1,190^{\mathrm{NS}}$ & $0,575^{\mathrm{NS}}$ & $0,515^{\mathrm{NS}}$ & $0,994^{\mathrm{NS}}$ \\
\hline $\mathrm{s}$ & 1,333 & 0,162 & 2,633 & 0,172 & 1,490 & 0,255 & 3,967 & 1,828 \\
\hline $\mathrm{CV}$ & 4,99 & 9,09 & 8,43 & 9,45 & 22,76 & 10,92 & 37,76 & 12,16 \\
\hline
\end{tabular}

Ns Ajuste não-significativo pelo teste $\mathrm{F}$, a $5 \%$ de probabilidade.

* Significativo a $5 \%$ de probabilidade, pelo teste F. 
TABELA 4 - Resumo da análise de variância para teores foliares de nutrientes em bananeira 'Prata-anã' (terceira folha amostrada na floração), no segundo ciclo de avaliação, em reposta à adubação com $\mathrm{B}$ e $\mathrm{Zn}$ (g família ${ }^{-1} a_{n o}^{-1}$ ). Epamig/CTNM, Jaíba-2006.

\begin{tabular}{|c|c|c|c|c|c|c|c|c|}
\hline & $\mathrm{N}$ & $P$ & $\mathrm{~K}$ & $S$ & $\mathrm{Ca}$ & $\mathrm{Mg}$ & \multirow{2}{*}{\multicolumn{2}{|c|}{ B $\mathrm{mg} \mathrm{kg}^{-1}$}} \\
\hline & \multicolumn{6}{|c|}{ - } & & \\
\hline & & & & & & & & \\
\hline 0 & 26,35 & 1,52 & 30,67 & 1,79 & 5,92 & 1,94 & 12,21 & 12,20 \\
\hline 2,5 & 26,36 & 1,51 & 30,24 & 1,78 & 5,77 & 1,86 & 10,88 & 12,65 \\
\hline 5,0 & 26,51 & 1,49 & 30,07 & 1,77 & 6,30 & 1,99 & 11,98 & 12,96 \\
\hline 7,5 & 26,69 & 1,52 & 30,60 & 1,74 & 5,94 & 1,97 & 11,41 & 12,52 \\
\hline 10,0 & 26,49 & 1,52 & 30,46 & 1,72 & 5,83 & 1,92 & 10,19 & 12,81 \\
\hline Teste F & $0,26^{\mathrm{NS}}$ & $0,35^{\mathrm{NS}}$ & $0,37^{\mathrm{NS}}$ & $0,58^{\mathrm{NS}}$ & $0,92^{\mathrm{NS}}$ & $1,55^{\mathrm{NS}}$ & $1,71^{\mathrm{NS}}$ & $0,92^{\mathrm{NS}}$ \\
\hline DMS $(5 \%)$ & 1,061 & 0,078 & 1,598 & 0,138 & 0,828 & 0,159 & 2,459 & 1,179 \\
\hline \multicolumn{9}{|l|}{ B } \\
\hline 0 & 26,20 & 1,52 & 30,80 & 1,79 & 6,03 & 1,96 & 10,12 & 12,88 \\
\hline 0,68 & 26,79 & 1,52 & 30,56 & 1,77 & 5,96 & 1,92 & 11,27 & 12,51 \\
\hline 1,36 & 26,48 & 1,50 & 30,44 & 1,71 & 6,09 & 1,94 & 11,84 & 12,25 \\
\hline 2,04 & 26,45 & 1,50 & 29,82 & 1,77 & 5,72 & 1,91 & 12,11 & 12,88 \\
\hline Teste F & $0,974^{\mathrm{NS}}$ & $0,438^{\mathrm{NS}}$ & $1,306^{\mathrm{NS}}$ & $1,184^{\mathrm{NS}}$ & $0,730^{\mathrm{NS}}$ & $0,357^{\mathrm{NS}}$ & $2,438^{\mathrm{NS}}$ & $1,300^{\mathrm{NS}}$ \\
\hline DMS $(5 \%)$ & 0,89 & 0,07 & 1,34 & 0,12 & 0,70 & 0,13 & 2,07 & 0,99 \\
\hline \multicolumn{9}{|l|}{ B x Zn } \\
\hline Teste F & $0,893^{\mathrm{NS}}$ & $1,692^{\mathrm{NS}}$ & $2,127^{*}$ & $0,775^{\mathrm{NS}}$ & $0,411^{\mathrm{NS}}$ & $0,339^{\mathrm{NS}}$ & 2,440 ** & $1,164^{\mathrm{NS}}$ \\
\hline $\mathrm{s}$ & 1,721 & 0,126 & 2,592 & 0,224 & 1,343 & 0,258 & 3,988 & 1,912 \\
\hline $\mathrm{CV}$ & 6,50 & 8,38 & 8,52 & 12,74 & $22,5 /$ & 13,34 & 35,19 & 15,14 \\
\hline
\end{tabular}

Ns Ajuste não-significativo pelo teste $\mathrm{F}$, a $5 \%$ de probabilidade.

* Significativo a $5 \%$ de probabilidade, pelo teste F.

** Significativo a $1 \%$ de probabilidade, pelo teste F.

TABELA 5 - Resumo da análise de variância para teores foliares de nutrientes em bananeira 'Prata-anã' (terceira folha amostrada na floração), no terceiro ciclo de avaliação, em reposta à adubação com $\mathrm{B}$ e $\mathrm{Zn}$ (g família ${ }^{-1}$ ano $^{-1}$ ). Epamig/CTNM, Jaíba - 2006.

\begin{tabular}{|c|c|c|c|c|c|c|c|c|}
\hline & $\mathrm{N}$ & $\mathrm{P}$ & $\mathrm{K}$ & $\mathrm{S}$ & $\mathrm{Ca}$ & $\mathrm{Mg}$ & $\mathrm{B}$ & $\mathrm{Zn}$ \\
\hline & -.---.- & -.-- & ------ g & $\mathrm{gg}^{-1}-$ & 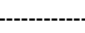 & 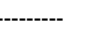 & ----- mg & $\mathrm{kg}^{-1}$---- \\
\hline $\mathrm{Zn}$ & & & & & & & & \\
\hline 0 & 24,58 & 1,52 & 29,44 & 1,60 & 4,86 & 1,99 & 10,66 & 13,09 \\
\hline 2,5 & 25,41 & 1,52 & 29,30 & 1,55 & 5,15 & 2,01 & 10,36 & 13,64 \\
\hline 5,0 & 25,44 & 1,50 & 29,62 & 1,59 & 5,13 & 2,08 & 10,41 & 13,38 \\
\hline 7,5 & 25,37 & 1,56 & 29,45 & 1,64 & 5,17 & 2,11 & 10,25 & 14,25 \\
\hline 10,0 & 24,58 & 1,53 & 29,92 & 1,56 & 4,61 & 1,92 & 10,13 & 13,60 \\
\hline Teste $\mathrm{F}$ & $2,130^{\mathrm{NS}}$ & $1,363^{\mathrm{NS}}$ & $0,387^{\mathrm{NS}}$ & $1,592^{\mathrm{NS}}$ & $2,771^{*}$ & $3,870^{* *}$ & $0,224^{\mathrm{NS}}$ & $1,960^{\mathrm{NS}}$ \\
\hline DMS $(5 \%)$ & 1,22 & 0,08 & 1,45 & 0,11 & 0,57 & 0,15 & 1,66 & 1,20 \\
\hline B & & & & & & & & \\
\hline 0 & 24,95 & 1,52 & 29,49 & 1,63 & 4,80 & 2,04 & 9,33 & 13,63 \\
\hline 0,68 & 25,02 & 1,51 & 29,40 & 1,50 & 5,05 & 2,00 & 11,55 & 13,57 \\
\hline 1,36 & 25,56 & 1,54 & 29,79 & 1,64 & 5,04 & 2,02 & 10,07 & 13,63 \\
\hline 2,04 & 24,77 & 1,52 & 29,57 & 1,58 & 5,05 & 2,26 & 10,49 & 13,53 \\
\hline Teste $\mathrm{F}$ & $1,498^{\mathrm{NS}}$ & $0,460^{\mathrm{NS}}$ & $0,252^{\mathrm{NS}}$ & $5,810^{* *}$ & $0,869^{\mathrm{NS}}$ & $0,221^{\mathrm{NS}}$ & $5,971^{* *}$ & $0,030^{\mathrm{NS}}$ \\
\hline DMS $(5 \%)$ & 1,02 & 0,07 & 1,22 & 0,10 & 0,48 & 0,12 & 1,39 & 1,01 \\
\hline B x Zn & & & & & & & & \\
\hline Teste $\mathrm{F}$ & $0,923^{\mathrm{NS}}$ & $0,868^{\mathrm{NS}}$ & $1,367^{\mathrm{NS}}$ & $2,438^{* *}$ & $0,756^{\mathrm{NS}}$ & $0,837^{\mathrm{NS}}$ & $3,577 * *$ & $0,955^{\mathrm{NS}}$ \\
\hline $\mathrm{s}$ & 1,972 & 0,133 & 2,352 & 0,187 & 0,933 & 0,239 & 2,685 & 1,939 \\
\hline $\mathrm{CV}$ & 7,87 & 8,72 & 7,96 & 11,80 & 18,72 & 11,80 & 25,91 & 14,27 \\
\hline
\end{tabular}

NS Ajuste não-significativo pelo teste $\mathrm{F}$, a $5 \%$ de probabilidade

* Significativo a $5 \%$ de probabilidade, pelo teste F.

** Significativo a $1 \%$ de probabilidade, pelo teste F.
TABELA 6 - Valores médios de teores foliares de K em bananeira 'Prata-anã' (terceira folha amostrada na floração), no segundo ciclo de avaliação, resultante da interação da adubação com B e Zn (g família $\left.{ }^{-1} \mathrm{ano}^{-1}\right)$. Epamig/CTNM, Jaíba-2006.

\begin{tabular}{lccccc}
\hline $\begin{array}{l}\mathrm{Zn} \\
\mathrm{B}\end{array}$ & 0 & 2,5 & 5,0 & 7,5 & 10,0 \\
\hline 0 & $32,84 \mathrm{~A} \mathrm{a}$ & $30,37 \mathrm{~A} \mathrm{ab}$ & $29,08 \mathrm{~A} \mathrm{~b}$ & $30,67 \mathrm{~A} \mathrm{ab}$ & $31,05 \mathrm{~A} \mathrm{ab}$ \\
0,68 & $31,36 \mathrm{~A} \mathrm{a}$ & $30,62 \mathrm{~A} \mathrm{a}$ & $29,90 \mathrm{~A}$ a & $29,63 \mathrm{~A} \mathrm{a}$ & $31,31 \mathrm{~A} \mathrm{a}$ \\
1,36 & $30,80 \mathrm{~A} \mathrm{a}$ & $30,12 \mathrm{~A} \mathrm{a}$ & $30,44 \mathrm{~A} \mathrm{a}$ & $31,26 \mathrm{~A} \mathrm{a}$ & $29,59 \mathrm{~A} \mathrm{a}$ \\
2,04 & $27,69 \mathrm{~B} \mathrm{a}$ & $29,86 \mathrm{~A} \mathrm{a}$ & $30,86 \mathrm{~A} \mathrm{a}$ & $30,82 \mathrm{~A} \mathrm{a}$ & $29,87 \mathrm{~A} \mathrm{a}$ \\
\hline $\mathrm{S}=0,259$ & & & & \\
$\mathrm{CV}=8,52$ & & & & \\
\hline
\end{tabular}

Médias seguidas pelas mesmas letras, maiúsculas na coluna ou minúsculas na linha, são iguais entre si, pelo teste de Tukey,a $5 \%$.

TABELA 7 - Valores médios de teores foliares de $\mathrm{S}$ em bananeira 'Prata-anã' (terceira folha amostrada na floração), no terceiro ciclo de avaliação, resultante da interação da adubação com B e Zn (g família $\left.{ }^{-1} \mathrm{ano}^{-1}\right)$. Epamig/CTNM, Jaíba-2006.

\begin{tabular}{lccccc}
\hline $\mathrm{Zn}$ & 0 & 2,5 & 5,0 & 7,5 & 10,0 \\
$\mathrm{~B}$ & & & & & \\
\hline 0 & $1,60 \mathrm{~A} \mathrm{ab}$ & $1,54 \mathrm{~A} \mathrm{~b}$ & $1,64 \mathrm{~A} \mathrm{ab}$ & $1,80 \mathrm{~A} \mathrm{a}$ & $1,56 \mathrm{AB} \mathrm{b}$ \\
0,68 & $1,62 \mathrm{~A} \mathrm{a}$ & $1,55 \mathrm{~A} \mathrm{ab}$ & $1,49 \mathrm{~A} \mathrm{ab}$ & $1,47 \mathrm{C} \mathrm{ab}$ & $1,36 \mathrm{~B} \mathrm{~b}$ \\
1,36 & $1,53 \mathrm{~A} \mathrm{a}$ & $1,62 \mathrm{~A} \mathrm{a}$ & $1,61 \mathrm{~A} \mathrm{a}$ & $1,75 \mathrm{AB} \mathrm{a}$ & $1,68 \mathrm{~A} \mathrm{a}$ \\
2,04 & $1,63 \mathrm{~A} \mathrm{a}$ & $1,48 \mathrm{~A} \mathrm{a}$ & $1,62 \mathrm{~A} \mathrm{a}$ & $1,55 \mathrm{BC} \mathrm{a}$ & $1,63 \mathrm{~A} \mathrm{a}$ \\
\hline $\mathrm{S}=0,187$ & & & & \\
$\mathrm{CV}=11,80$ & & & & \\
\hline
\end{tabular}

Médias seguidas pelas mesmas letras, maiúsculas na coluna ou minúsculas na linha, são iguais entre si, pelo teste de Tukey, a 5\%.

TABELA 8 - Resumo da análise de variância para efeito da aplicação de Zn e B (g família ${ }^{-1}$ ano $^{-1}$ ), na massa de frutos por cacho $(\mathrm{kg})$ de bananeira 'Prata-anã', em três ciclos de avaliação. Epamig/CTNM, Jaíba 2006.

\begin{tabular}{|c|c|c|c|}
\hline & \multicolumn{3}{|c|}{ Massa de frutos por cacho $(\mathrm{kg})$} \\
\hline & Ciclo 1 & Ciclo 2 & Ciclo 3 \\
\hline \multicolumn{4}{|l|}{ Zn } \\
\hline & 28,99 & $33,13 \mathrm{~A}$ & $31,68 \mathrm{~A}$ \\
\hline 2,5 & 29,58 & $32,77 \mathrm{AB}$ & $31,17 \mathrm{AB}$ \\
\hline 5,0 & 28,16 & $29,70 \mathrm{~B}$ & $29,44 \mathrm{AB}$ \\
\hline 7,5 & 27,61 & $30,86 \mathrm{~B}$ & $28,67 \mathrm{~B}$ \\
\hline 10,0 & 27,92 & $31,29 \mathrm{AB}$ & 30,98 AB \\
\hline Teste F & $0,926^{\mathrm{NS}}$ & $3,115 *$ & $2,867 *$ \\
\hline DMS $(5 \%)$ & 3,26 & 3,08 & 2,90 \\
\hline \multicolumn{4}{|l|}{$\mathbf{B}$} \\
\hline $\mathbf{O}$ & 28,52 & 31,67 & 29,44 \\
\hline 0,68 & 28,97 & 31,20 & 30,68 \\
\hline 1,36 & 28,64 & 31,95 & 30,97 \\
\hline 2,04 & 27,69 & 31,35 & 30,47 \\
\hline Teste F & $0,522^{\mathrm{NS}}$ & $0,221^{\mathrm{NS}}$ & $0,979^{\mathrm{NS}}$ \\
\hline DMS $(5 \%)$ & 2,74 & 2,59 & 2,44 \\
\hline $\mathrm{B} \times \mathrm{Zn}$ & $0,462^{N S}$ & $0,500^{N S}$ & $0,988^{\mathrm{NS}}$ \\
\hline $\mathbf{s}$ & 5,341 & 5,054 & 4,753 \\
\hline CV & $18,7 /$ & 16,02 & 15,64 \\
\hline
\end{tabular}

Ns Ajuste não-significativo pelo teste $\mathrm{F}$, a $5 \%$ de probabilidade.

* Significativo a $5 \%$ de probabilidade, pelo teste F.

Médias seguidas pela mesma letra na coluna (no caso de $\mathrm{F}$ significativo) não diferem entre si, pelo teste de Tukey (5\%). 

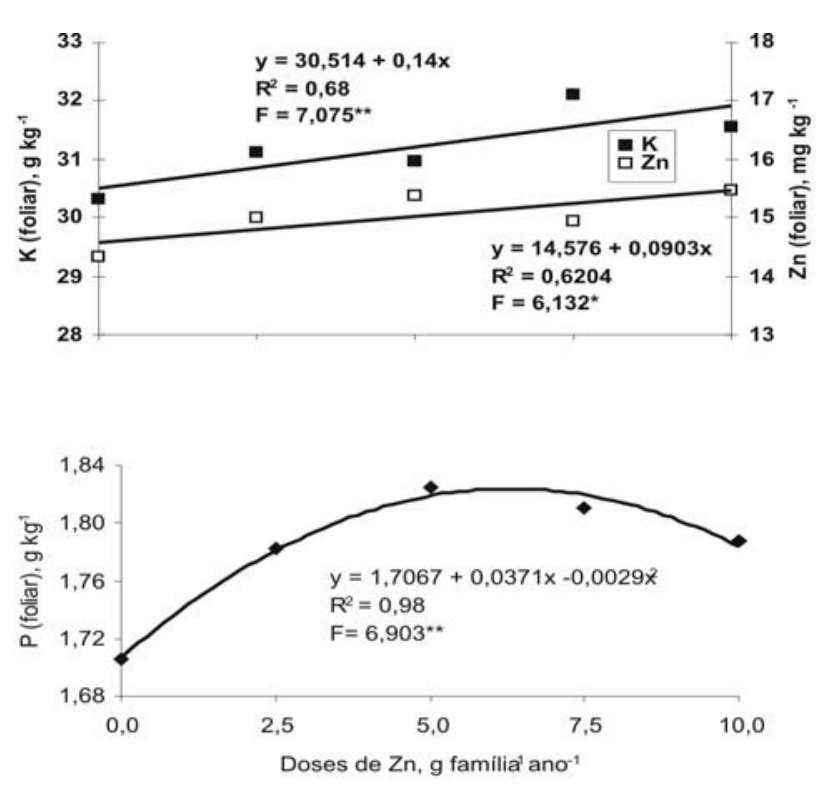

FIGURA 1- Teores foliares de potássio, zinco e fósforo em bananeira 'Prata-anã' (terceira folha amostrada na floração), em resposta à aplicação de zinco, no primeiro ciclode avaliação. Epamig/CTNM,Jaíba-2006.

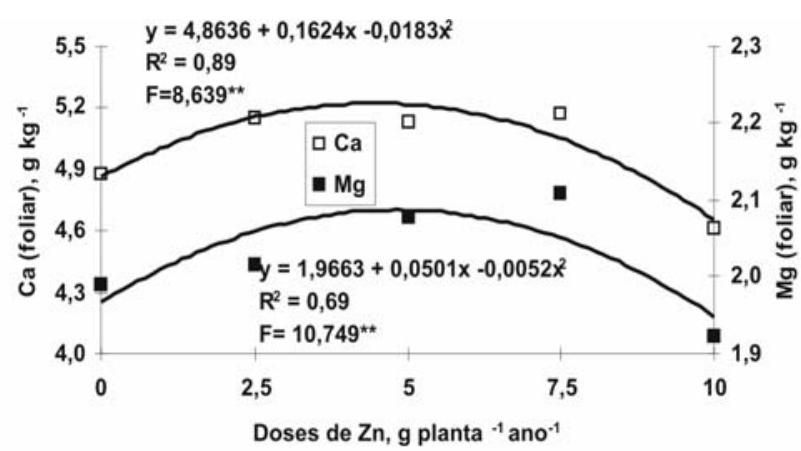

FIGURA 2 - Teores foliares de cálcio e magnésio em bananeira 'Prata- anã' (terceira folha amostrada na floração), em resposta à aplicação de zinco, no terceiro ciclo de avaliação. Epamig/CTNM, Jaíba - 2006.

\section{CONCLUSÕES}

1- As doses de $\mathrm{Zn}$ e B fornecidas diretamente no rizoma da bananeira, via muda desbastada, alteraram os teores foliares de nutrientes (P,K e Zn no primeiro ciclo; K e B no segundo ciclo. S, $\mathrm{Ca}, \mathrm{Mg}$ e B no terceiro ciclo), porém, sem magnitude capaz de afetar a condição nutricional quando se consideram as faixas de suficiência.

2- O fornecimento de nutrientes diretamente no rizoma não supriu a demanda das plantas em $\mathrm{Zn}$, nas doses utilizadas, uma vez que estas apresentaram deficiência do elemento no segundo e terceiro ciclos avaliados. Para B, houve efeito positivo, apesar de não ser acentuado.
3- A aplicação de Zn propiciou menor massa de frutos por cacho nas doses intermediárias, embora essa resposta não se adapte a um padrão biológico.

4- Os resultados obtidos indicam que há necessidade de novas pesquisas para se obterem maiores informações sobre uma possível diferença de translocação do $\mathrm{Zn}$ entre cultivares do sbgrupo Cavendish e a Prata-anã.

\section{REFERÊNCIAS}

ABANORTE - ASSOCIAÇÃO CENTRAL DOS FRUTICULTORES DO NORTE DE MINAS. Banana tipo exportação. Janaúba; 2005. Disponível em: <www.abanorte.com.br/pesquisa_inovacao/ padronizacao_banana>. Acesso: 5 jun. 2006.

ALVAREZ V.V.H.; NOVAIS, R. F. de; BARROS, N. F. de; CANTARUTTI, R. B.; LOPES, A.S. Interpretação dos resultados das análises de solos. In: RIBEIRO, A. C.; GUIMARÃES, P. T. G.; ALVAREZ V., V. H. Recomendações técnicas para o uso de corretivos e fertilizantes em Minas Gerais: 5a aproximação. Viçosa: CFSEMG, 1999. p. 25-32.

BLANCHAR, R. W., REHM, G., CALDWELL, A. /C. Sulfur in plant material by degestion with nitric and perchloric acid. Soil Science Society American Proceedings, Madison, v. 29, n. 1, p.71-72, 1965 .

BORGES, A. L.; OLIVEIRA, A. M. G.; SOUZA, L. S. Solos, nutrição e adubação. In: ALVES, E. J. (Coord.). A cultura da banana: aspectos técnicos, socioeconômicos e agroindustriais. Brasília: Embrapa- SPI, 1987.p. 197-260.

BRAGA, J.M., DEFELIPO, B.V. Determinação espectrofotométrica de fósforo em extratos de solos e plantas._Revista Ceres, Viçosa, v.21, p. 73-85, 1974.

EMPRESA BRASILEIRA DE PESQUISA AGROPECUÁRIA. Centro Nacional de Pesquisa de Solos. Manual de métodos de análise do solo. Brasília: SPI, 1997. 212 p.

LÓPEZ, A.; ESPINOSA, J. Manual de nutricion y fertilización del banano. Quito: INPOFOS, 1995. $82 \mathrm{p}$.

MALAVOLTA, E. Elementos de nutrição mineral de plantas. São Paulo: Agronômica Ceres, 1980.251 p.

MALAVOLTA, E.; VITTI, G. C.; OLIVEIRA, S. A.de. Avaliação do estado nutricional das plantas. Piracicaba: Potafós, 1997. 319 p.

MALBURG, J. L. Métodos de amostragem foliar para a diagnose nutricional da bananeira 'Enxerto' ('Prata anã') no sul de Santa 
Catarina. 1988. 94 f. Dissertação (Mestrado em agronomia) Escola Superior de Agricultura de Lavras, Lavras, 1988.

MARSCHNER, H. Mineral nutrition of higher plants. $2^{\text {nd }}$ ed. London: Academic press, $1995.889 \mathrm{p}$.

MARTIN-PRÉVEL, P. Banana. In: MARTIN-PRÉVEL, P.; GAGNARD, J.; GAUTIER, P. (Ed.). Plant analysis: as a guide to the nutrient requirements of temperature and tropical crops. Paris: Lavoisier Publising, 1987. p. 637-676.

MENGUEL, K.; KIRKBY, E. A. Principles of plant nutrition. $4^{\text {th }}$ ed. Bern: International potash Institute, 1987. 387p.

MOREIRA, R. S. Banana: teoria e prática de cultivo. São Paulo: Fundação Cargill, 1999. 1 CD-ROM.

MOREIRA, R. S. Adubação. In: SIMPÓSIO BRASILEIRO SOBRE BANANICULTURA, 4., 1998, Campo Grande. Anais... Jaboticabal: UNESP, 2001. p. 252-325.

PINHO, P. J. de. Nutrição mineral, produtividade e classificação dos frutos de bananeira 'Prata anã' irrigada do norte de Minas Gerais sob aplicação de zinco no solo. 2004. 64 f. Dissertação (Mestrado em Agronomia) - Universidade Federal de Lavras, Lavras, 2004.

PREZOTTI, L. C. Recomendações de calagem e adubação para o Estado do Espírito Santo: $3^{\mathfrak{a}}$ aproximação. Vitória: EMCAPA, 1992. 73 p. (Circular Técnica, 12).

QUAGGIO, J. A.; PIZZA JUNIOR, C. T. Frutíferas tropicais. In: FERREIRA, M. E.; CRUZ, M. C. P. da; RAIJ, B. van; ABREU, C. A. de. (Ed.). Micronutrientes e elementos tóxicos na agricultura.
Jaboticabal: CNPq/ FAPESP/ POTAFOS, 2001. p. 459-492.

SILVA, E. de B.; RODRIGUES, M. G. V. Levantamento nutricional dos bananais da região norte de Minas Gerais pela análise foliar. Revista Brasileira de Fruticultura, Jaboticabal, v. 23, n. 3, p. 695-698, 2001.

SILVA, J.T.A. da; BORGES, A.L.; MALBURG, J.L. Solos, adubação e nutrição da bananeira. Informe Agropecuário, Belo Horizonte, v.20, n.196, p.21-36, 1999.

SILVA, J.T.A. da; BORGES, A.L.; DIAS, M. S. C.; COSTA, E. L. da; PRUDÊNCIO, J. M. Diagnóstico nutricional da bananeira 'Prata anã' para o norte de Minas. Belo Horizonte: EPAMIG, 2002.16 p. (Boletim Técnico, 70).

SOTO BALLESTERO, M. Bananos: cultivo y comercialización. $2^{\text {nd }}$ ed. San José: Imprenta Lil, 2000. 1 CD-ROM.

TEDESCO, M.J.; VOLKWEISS, S.J.; BOHNEN, H. Análises de solo, plantas e outros materiais. Porto Alegre: Departamento de Solos, Faculdade de Agronomia, UFRS, 1985. 186p. (Boletim Técnico, 5).

TEIXEIRA, L.A.; SPIRONELLO, A.; QUAGGIO, J.A.; FURLANI, P. R. Banana. In: RAIJ, B. van; CANTARELLA, H., QUAGGIO, J. A.; FURLANI, A. M. C. (Ed.). Recomendações de adubação e calagem para o Estado de São Paulo. 2. ed. Campinas: Instituto Agronômico/Fundação IAC, 1997.p. 131-132. (Boletim Técnico, $100)$ 